\title{
Clinical Observations Completion Status
}

National Cancer Institute

\section{Source}

National Cancer Institute. Clinical Observations Completion Status. NCI Thesaurus. Code C119812.

A term used to describe the state or condition of the completeness of the clinical observation data. 